\section{AWE-10 AN INTERNATIONAL SURVEY ON THE DIAGNOSIS AND MANAGEMENT OF SEVERE GASTROINTESTINAL DYSMOTILITY}

${ }^{1}$ Dipesh H Vasant* ${ }^{2}$ Loris Pironi, ${ }^{3}$ Frederico Bozzetti, ${ }^{4}$ Cristina Cuerda, ${ }^{5}$ Francisca Joly, ${ }^{6}$ Darlene Kelly, ${ }^{7}$ Peter Paine, ${ }^{7}$ Michael Staun, ${ }^{8}$ Kinga Szczepanek, ${ }^{9}$ André Van Gossum, ${ }^{10}$ Geert Wanten, ${ }^{11}$ Simon Lal. ${ }^{1}$ Manchester University Foundation Trust, Manchester, UK; ${ }^{2}$ University of Bologna, Bologna, Italy; ${ }^{3}$ University of Milan, Milan, Italy; ${ }^{4}$ HGGM Nutrition Unit, Madrid, Spain; ${ }^{5} \mathrm{MICl}$ et Assistance Nutritive, Clichy, France; ${ }^{6}$ Mayo Clinic, Rochester, USA; ${ }^{7}$ Salford Royal Foundation Trust, UK; ${ }^{8}$ University of Copenhagen, Copenhagen, Denmark; ${ }^{9}$ Stanley Dudrick's Memorial Hospital, Skawina, Poland; ${ }^{10}$ Erasme Hospital, Brussels, Belgium; ${ }^{11}$ Radboud university medical center, Nijmegen, Netherlands

\subsection{6/gutjnl-2019-BSGAbstracts.395}

Introduction Severe gastrointestinal dysmotility (GID) can be sub-classified into Chronic Intestinal Pseudo-obstruction (CIPO) and Enteric Dysmotility (ED) subtypes. We surveyed current opinions on the diagnosis and management of GID amongst experts from different countries.

Methods An survey questionnaire developed by the European society for Clinical Nutrition and Metabolism (ESPEN) was circulated electronically to members of ESPEN, European Society of Neurogastroenterology and Motility, and United European Gastroenterology. Only participants that completed all required components were included in the analysis.

Results Of 154 included participants, 82\% were European, the majority were attending clinicians/professors (85\%), based at either national/regional referral centres and/or academic institutions (87\%). Almost all (93\%) agreed that CIPO and ED should be classed separately. Most (73\%), reported increased incidence of GID, with $69 \%$ reporting an increase in ED. GID associated with hypermobile Ehlers-Danlos Syndrome was the group with the largest increase in referrals (37\%), however this trend was driven by observations from UK participants only $(\mathrm{P}<0.0001)$. Almost all clinicians $(95 \%)$ find diagnosing GID difficult, with 57\% finding ED more challenging and 32\% find both types equally difficult. GID diagnosis is often delayed (CIPO: by $>5$ years according to $16 \%$; ED: by $>5$ years according to $19 \%)$. Moreover, by the time of diagnosis, $>10 \%$ of patients have had inappropriate operations according to $82 \%$ of clinicians. Small Bowel Manometry, a test mandated to diagnose $\mathrm{ED}$, is surprisingly never used by $44 \%$, and is only used in $>50 \%$ cases by $21 \%$. Full thickness biopsies are usually requested from planned/previous resections $(33 \%)$ or when the diagnosis is unclear $(43 \%)$, but seldom change medical treatment (12\%), nutritional management $(16 \%)$ and prognosis (25\%). Very few treatments are useful for $>50 \%$ of patients, with antibiotics for SIBO, prucalopride, and psychology felt to be the most useful. Parenteral Nutrition (PN) rarely leads to improvement in symptoms (28\%), and is associated with PN dependency at 5-years according to the majority (56\%).

Conclusion These data highlight the difficulties with diagnosing and managing GID, even in 'expert' hands, and inform the urgent need for international, multidisciplinary, clinical practice guidelines.

\section{Posters}

\section{PWE-076 EFFICACY OF PHARMACOLOGICAL THERAPIES IN PATIENTS WITH IRRITABLE BOWEL SYNDROME WITH DIARRHOEA: NETWORK META-ANALYSIS}

\begin{abstract}
${ }^{1,2}$ Christopher Black*, ${ }^{1,2}$ Nicholas Burr, ${ }^{3}$ Michael Camilleri, ${ }^{4}$ David Earnest, ${ }^{5}$ Eamonn Quigley, ${ }^{6}$ Paul Moayyedi, ${ }^{2}$ Lesley Houghton, ${ }^{1,2}$ Alexander Ford. 'Leeds Gastroenterology Institute, SJUH, Leeds, UK; ${ }^{2}$ Leeds Institute of Medical Research at St James's, University of Leeds, Leeds, UK; ${ }^{3}$ Clinical Enteric Neuroscience Translational and Epidemiological Research, Mayo Clinic, Rochester, USA; ${ }^{4}$ Division of Gastroenterology, The University of Arizona College of Medicine, Tucson, USA; ${ }^{5}$ Division of Gastroenterology and Hepatology, Houston Methodist Hospital, Houston, USA; ${ }^{6}$ Gastroenterology Division, McMaster University, Hamilton, Canada
\end{abstract}

\subsection{6/gutjnl-2019-BSGAbstracts.396}

Introduction Over half of patients with irritable bowel syndrome have either diarrhoea (IBS-D) or a mixed stool pattern (IBS-M). The relative efficacy of licensed pharmacological therapies in IBS-D and IBS-M is unclear in the absence of head-to-head trials. We conducted a network meta-analysis to resolve this uncertainty.

Methods We searched MEDLINE, EMBASE, EMBASE Classic, the Cochrane central register of controlled trials, and clinicaltrials.gov through November 2018 to identify randomised controlled trials (RCTs) assessing the efficacy of licensed pharmacological therapies in adults with IBS-D or IBS-M. Trials included in the analysis reported a dichotomous assessment of overall response to therapy, and data were pooled using a random effects model. Efficacy and safety of all pharmacological therapies were reported as a pooled relative risk of remaining symptomatic with $95 \%$ confidence intervals (CIs) to summarise the effect of each comparison tested. Treatments were ranked according to their P-score.

Results We identified 18 eligible RCTs ( 7 alosetron, 5 ramosetron, 2 rifaximin, 4 eluxadoline), containing 9844 patients. All were superior to placebo for the treatment of IBS-D or IBS$\mathrm{M}$ at 12 weeks, according to the Food and Drug Administration (FDA)-recommended endpoint for trials in IBS (Figure 1). Alosetron 1mg twice-daily ranked first for efficacy, based on the FDA-recommended composite endpoint of improvement in both abdominal pain and stool consistency $(\mathrm{RR}=0.69 ; 95 \%$ CI 0.60 to 0.80 , P-score $=0.97$ ), effect on global symptoms of IBS, and effect on stool consistency. Ramosetron $2.5 \mathrm{mcg}$ once-daily was ranked first for effect on abdominal pain (RR $=0.75 ; 95 \%$ CI 0.65 to 0.85 , P-score $=0.94)$. Total numbers of adverse events were significantly greater with alosetron $1 \mathrm{mg}$ twice-daily and ramosetron $2.5 \mathrm{mcg}$, once-daily, compared with placebo. Rifaximin $550 \mathrm{mg}$ three times daily ranked first for safety. Constipation was significantly more common with all drugs, except rifaximin 550mg three times daily.

Conclusions In a network meta-analysis of randomised controlled trials of pharmacological therapies for IBS-D and IBS$\mathrm{M}$, we found all drugs to be superior to placebo, but alosetron and ramosetron appeared to be the most effective. 